\section{Resonant tunneling diode photodetectors for optical communications}

\author{
Scott Watson ${ }^{1}$ ( ) I Weikang Zhang ${ }^{1}$ । \\ Joana Tavares $^{2}$ (1) । Jose Figueiredo ${ }^{3}$ । \\ Horacio Cantu $^{4}$ | Jue Wang ${ }^{1}$ । \\ Edward Wasige $^{1}$ | Henrique Salgado ${ }^{2}$ । \\ Luis Pessoa $^{2}$ । Anthony Kelly ${ }^{1}$ \\ ${ }^{1}$ School of Engineering, University of Glasgow, Glasgow, United Kingdom \\ ${ }^{2}$ INESC TEC and Faculty of Engineering, University of Porto, Porto, \\ Portugal \\ ${ }^{3}$ Department of Physics, Faculty of Sciences, University of Lisbon, Lisbon, \\ Portugal \\ ${ }^{4}$ Compound Semiconductor Technologies Global Ltd, Glasgow, United \\ Kingdom
}

\section{Correspondence}

Scott Watson, School of Engineering, University of Glasgow, Rankine Building, Oakfield Avenue, Glasgow, G12 8LT, United Kingdom.

Email: scott.watson@glasgow.ac.uk

\section{Funding information}

European Union's Horizon 2020 Research and Innovation Programme, Grant/Award Number: 645369
Abstract
Optical modulation characteristics of resonant tunneling diode photodetectors (RTD-PD) are investigated. Inten- sity modulated light excites the RTD-PDs to conduct data experiments. Simple and complex data patterns are used with results showing data rates up to 80 and $200 \mathrm{Mbit} / \mathrm{s}$, respectively. This is the first demonstration of complex modulation using resonant tunneling diodes.

\section{KEYWORDS}
optical communications, photodetector, resonant tunneling diode, complex data transmission

\section{1 | INTRODUCTION}

The demand for high speed wired and wireless communications is ever-growing and it is expected that data rates in the order of tens of Gbit/s will be desired in the near future. ${ }^{1}$ In order to exploit higher frequency bands, the millimeter-wave and terahertz part of the spectrum are being considered. The

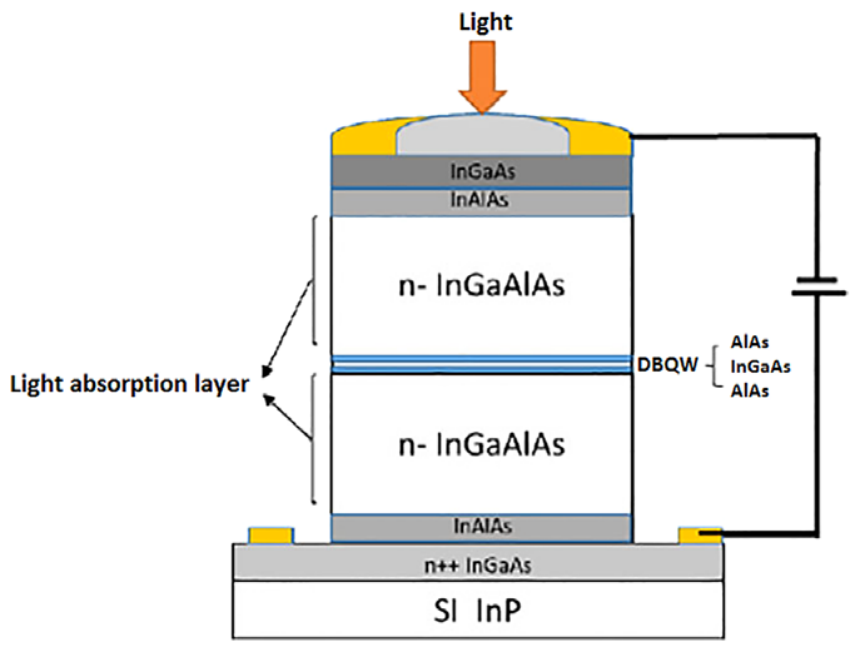

FIGURE 1 RTD-PD epi-layer structure [Colour figure can be viewed at wileyonlinelibrary.com]

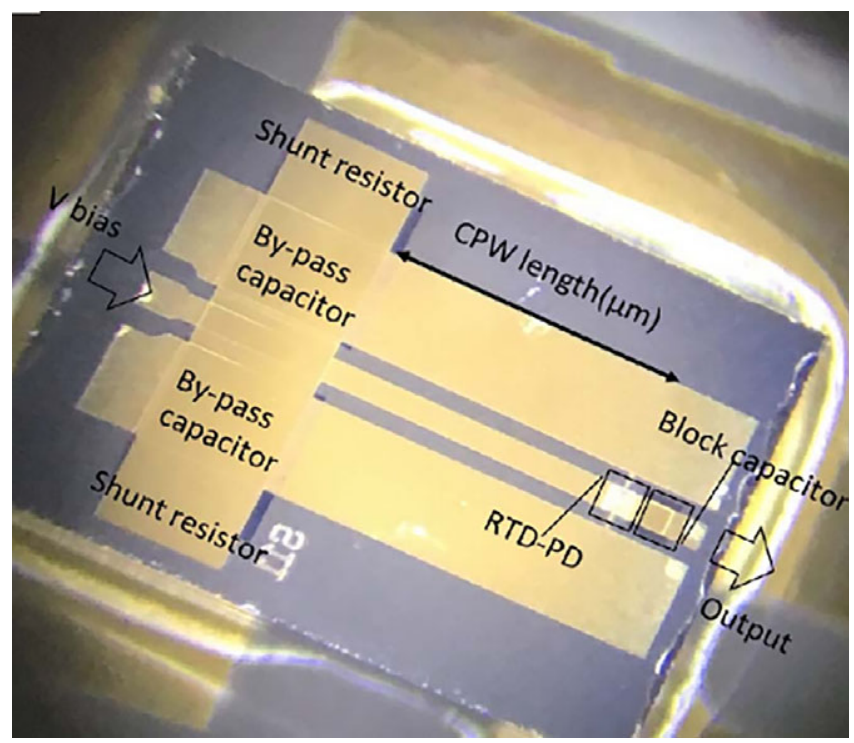

FIGURE 2 Photo of RTD-PD oscillator with oscillation frequency of $35 \mathrm{GHz}$ [Colour figure can be viewed at wileyonlinelibrary.com] 


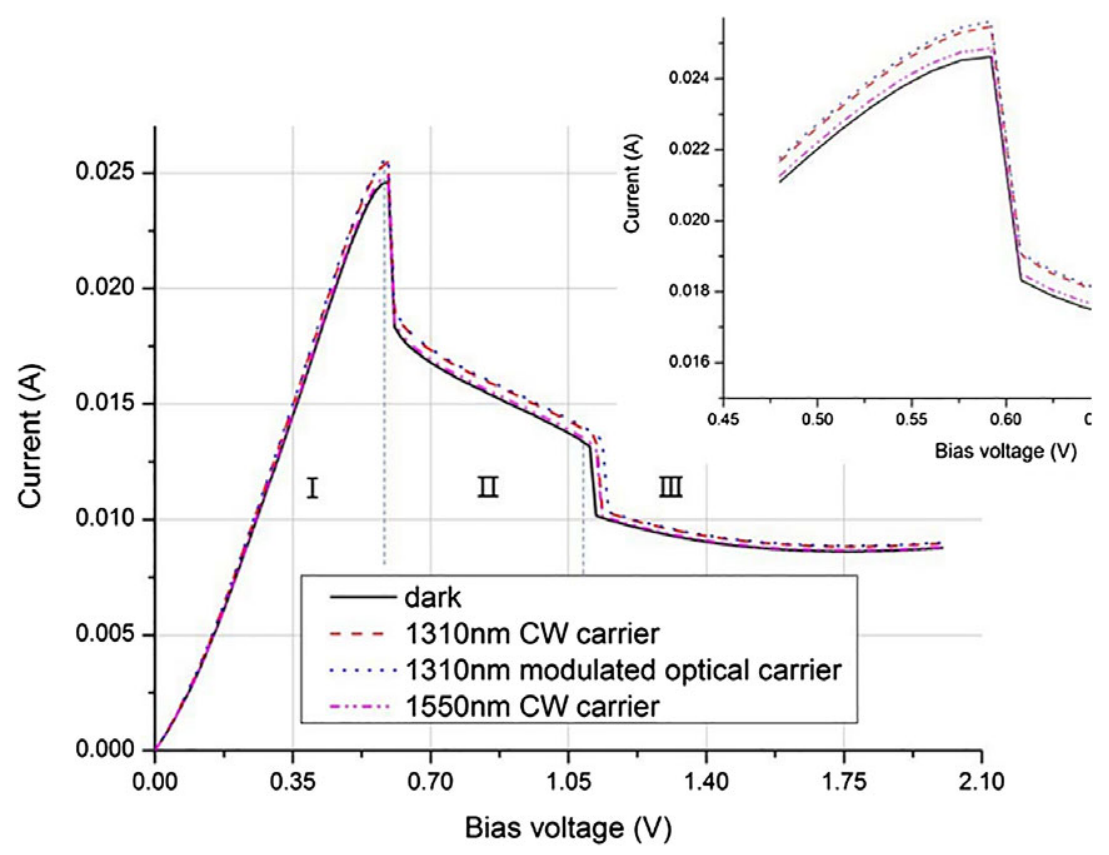

FIGURE 3 Measured I-V characteristic of the RTD-PD. Inset: The I-V curve shifting to the right with the presence of light [Colour figure can be viewed at wileyonlinelibrary.com]

lack of practical sources has been a bottleneck for this work but there are now a number of devices able to generate high frequency carrier waves suitable for communications into the terahertz bands. One example is resonant tunneling diodes (RTDs) which have the advantage of being potentially very low cost. RTDs have been the subject of research for many years resulting in usable output powers and oscillation frequencies up to $1.9 \mathrm{THz}^{2}$ RTDs are compact structures, of less than a millimeter square, implemented on monolithic microwave integrated circuit (MMIC) technology and have been demonstrated in systems using electrical data modulation up to $34 \mathrm{Gbit} / \mathrm{s}$ with carrier frequencies of approximately $500 \mathrm{GHz} .{ }^{3}$ It is possible to integrate RTDs with antennae and other optoelectronic components such as photodiodes. Resonant tunneling diode photodetectors (RTD-PDs) have a number of potential uses such as the

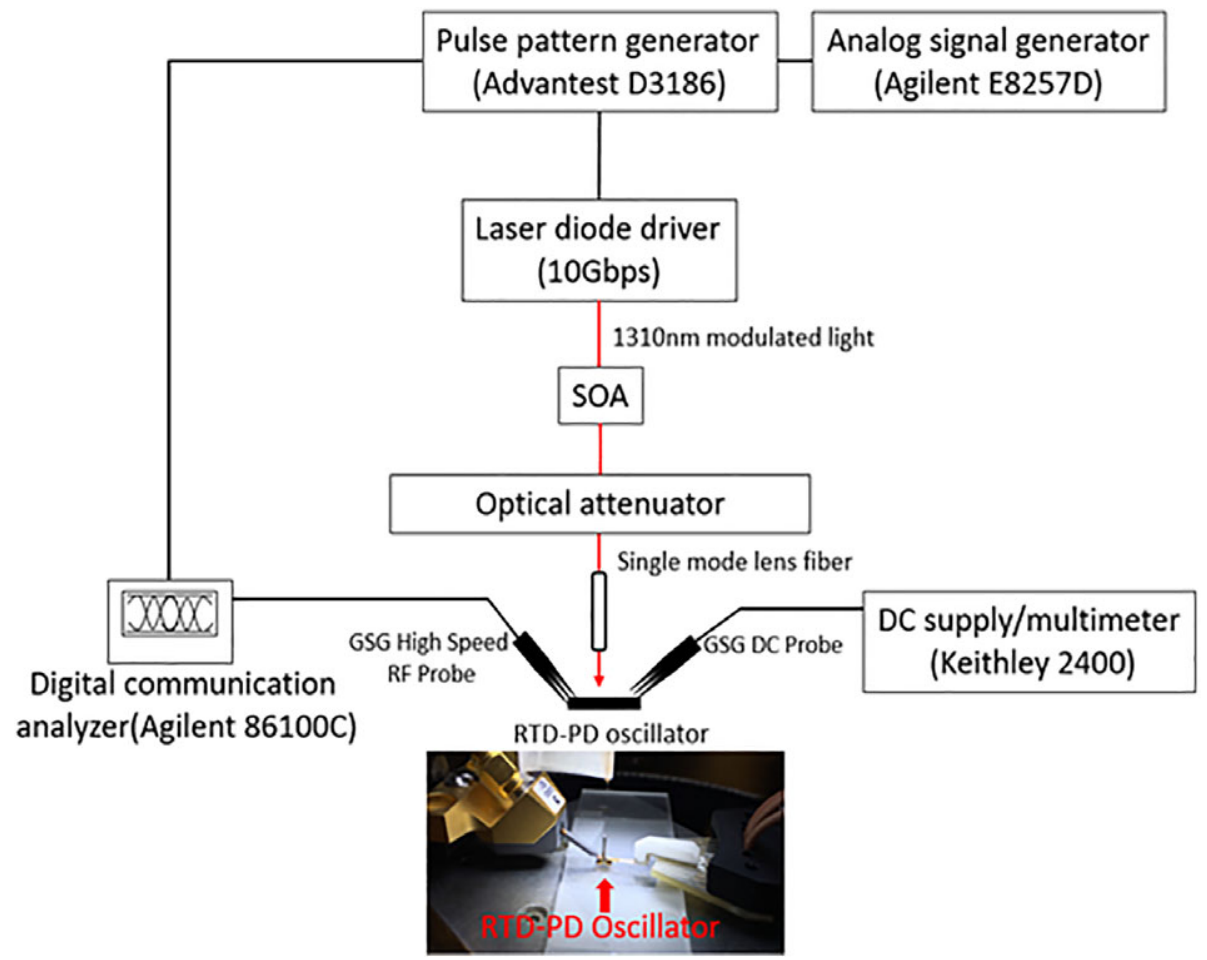

FIGURE 4 Experimental setup for optical modulation experiments [Colour figure can be viewed at wileyonlinelibrary.com] 


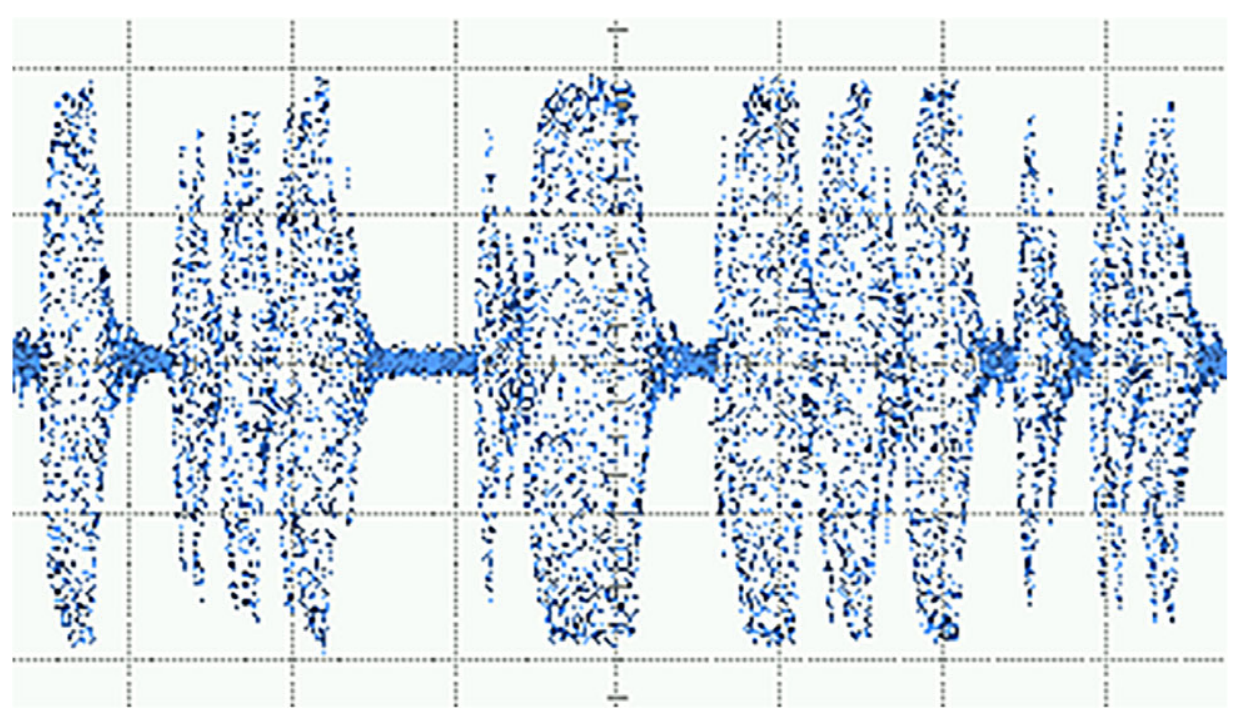

FIGURE 5 Output signal from the RTD-PD showing data patterns at $80 \mathrm{Mbit} / \mathrm{s}$ mixed with the oscillations [Colour figure can be viewed at wileyonlinelibrary.com]

seamless transferal of optical data onto an RF carrier and in fiber distributed networks as a means for optical synchronization of the generated RF carrier via injection locking.

This work discusses the performance of a range of RTDPDs. Optical to RF data transferral experiments is demonstrated. The use of complex modulation formats is also explored.

\section{I DEVICE STRUCTURE AND PERFORMANCE}

Figure 1 shows the epi-layer structure of an RTD-PD and Figure 2 shows a microscope image of the device. These
RTD-PDs have an optical window fabricated allowing direct access for the optical signal to reach the oscillator. As seen from the structure, they also include light absorption layers on both sides of the double barrier quantum well (DBQW), which increases the responsivity to light. More fabrication details can be found in previous work. ${ }^{4,5}$

Firstly, an I-V measurement was taken to establish the negative differential resistance (NDR) region and this was carried out in both light and dark conditions. The RTD-PD is sensitive to both 1310 and $1550 \mathrm{~nm}$; however, it can be seen in Figure 3 that the photocurrent is higher for $1310 \mathrm{~nm}$ due to the material used for the optical absorption layer.

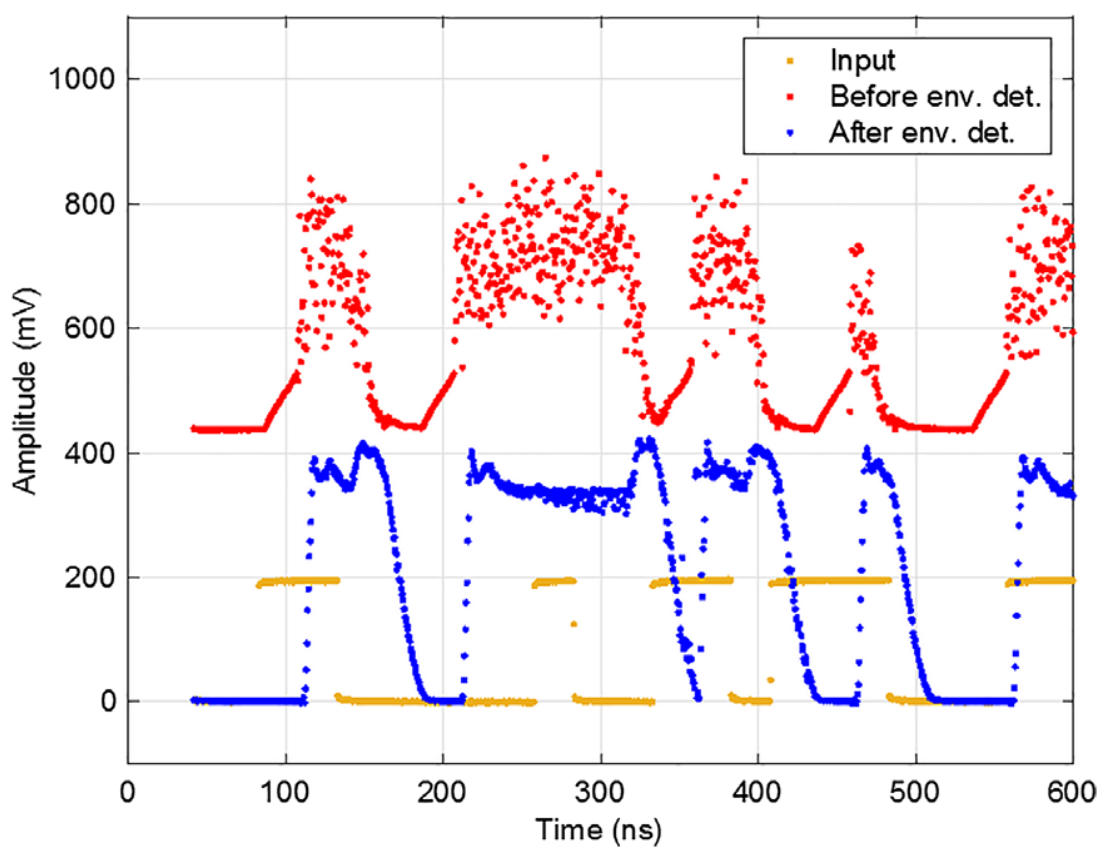

FIGURE 6 Measured time domain signals at the input (yellow), before (red), and after (blue) the envelope detector [Colour figure can be viewed at wileyonlinelibrary.com] 


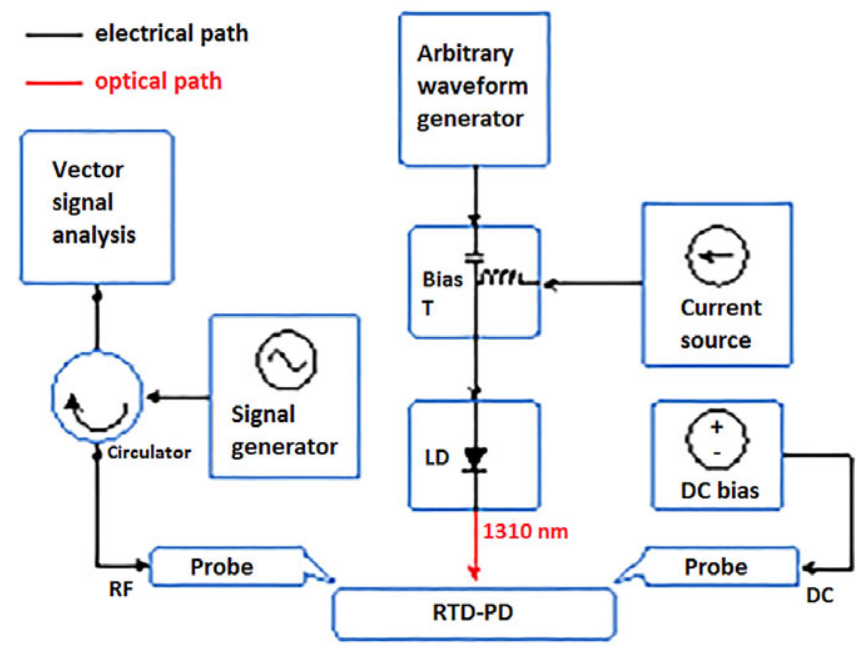

FIGURE 7 Experimental setup for advanced modulation formats evaluation [Colour figure can be viewed at wileyonlinelibrary.com]

\section{3 | DATA TRANSMISSION EXPERIMENTS}

Optical data transmission experiments were carried out using an RTD-PD with an oscillation frequency of $35 \mathrm{GHz}$. The oscillation frequency could be tuned by a few tens of megahertz by tuning the voltage, but also by introducing different powers of light incident on the oscillator. A pseudo-random bit sequence (PRBS) is used to modulate the optical signal and is sent to the oscillator via optical fiber, as seen in Figure 4. The output time domain signal was viewed on a fast oscilloscope so that the oscillations could be seen. Figure 5 shows the received pattern at $80 \mathrm{Mbit} / \mathrm{s}$, where an oscillation relates to a "1" bit and no oscillation is a "0" bit.
Higher data rates are expected in future work and can be achieved by optimizing the device structure for optical signals as well as improving the cut-off frequency set by the external circuitry.

Using an envelope detector at the output of the RTD-PD we were able to demodulate the transmitted signal, up to $40 \mathrm{Mbit} / \mathrm{s}$, which was limited by the envelope detector bandwidth (Figure 6).

Using another RTD-PD with an oscillation frequency around $14 \mathrm{GHz}$, complex data transmission measurements were conducted.

The implemented setup used to explore complex modulation is shown in Figure 7. The complex modulations are generated by an arbitrary waveform generator (AWG) that sends a subcarrier modulated complex signal through the DC port of the RTD. The output of the RTD is received by a spectrum analyzer running a vector signal analysis (VSA) software, used for evaluating the quality of the transmitted signal. A signal generator was used on the RF port side in order to lock the RTD oscillation carrier, which allows the impact of the RTD carrier phase noise to be assessed.

The signal-to-noise ratio (SNR) of the demodulated signals for both locked and unlocked regimes are shown in Figure 8 , as a function of the subcarrier frequency. In this experiment the AWG was generating 100 Mbaud QPSK signals. This result shows that for subcarrier frequencies below approximately $250 \mathrm{MHz}$, locking the RTD-PD carrier provides a considerable advantage, while above this value the locking does not significantly improve the results, which shows that the RTD-PD phase noise affects mainly the lower subcarrier frequencies due to the RTD-PD carrier

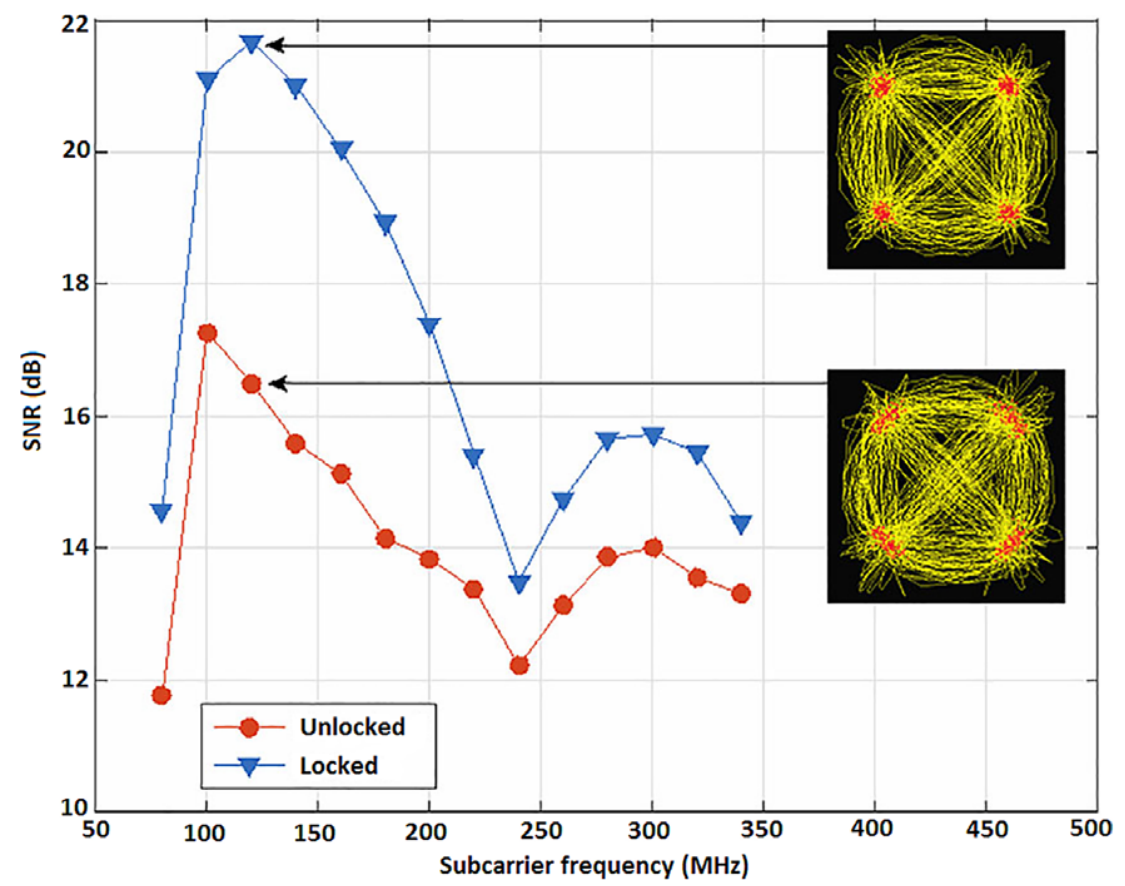

FIGURE 8 SNR as a function of subcarrier frequency measured for 100 MBaud QPSK data transmission. Inset: Constellation of the received signals (at $120 \mathrm{MHz}$ ) [Colour figure can be viewed at wileyonlinelibrary.com] 


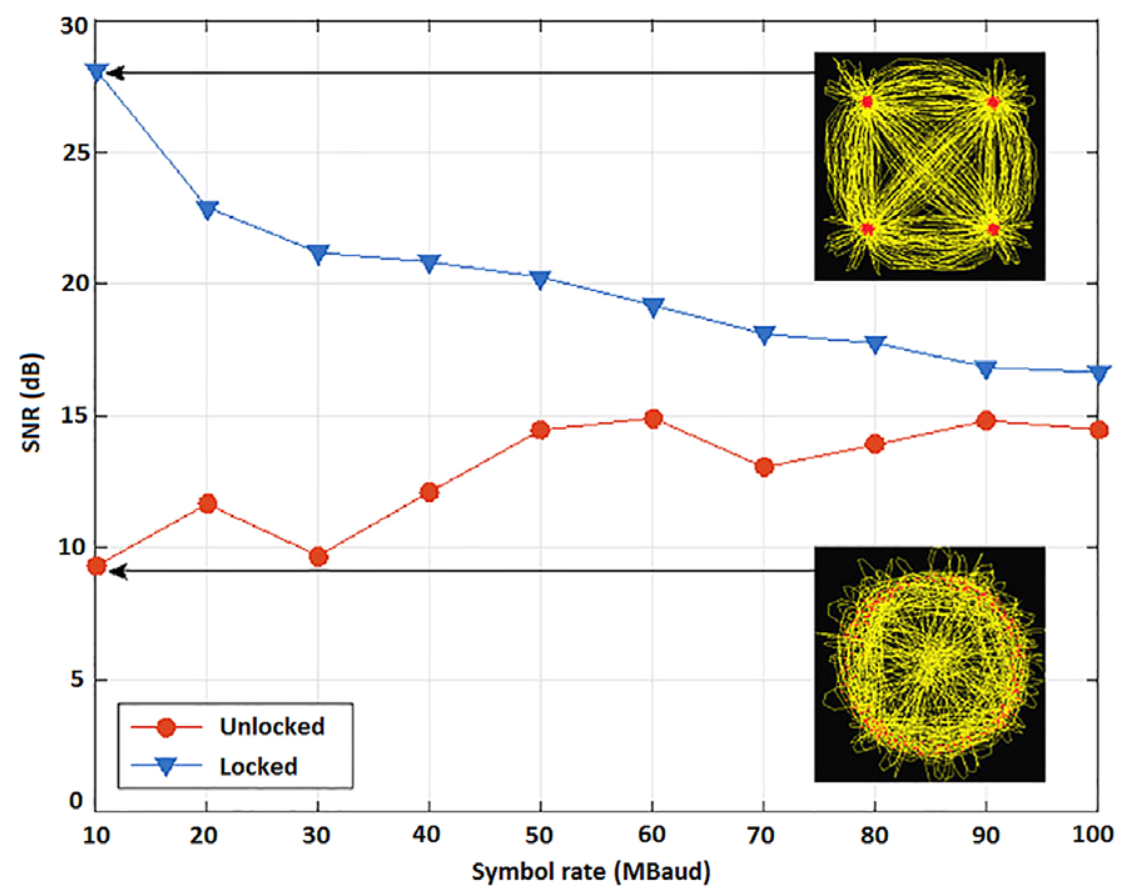

FIGURE 9 SNR as a function of symbol rate measured for a subcarrier frequency of $200 \mathrm{MHz}$. Inset: Constellation of the received signals (for $10 \mathrm{MBaud}$ ) [Colour figure can be viewed at wileyonlinelibrary.com]

proximity. The inset shows the constellation of the demodulated signals for a subcarrier frequency of $120 \mathrm{MHz}$, where the phase noise is visible in the constellation rotation when unlocked.

Figure 9 shows the SNR as a function of the symbol rate measured for a subcarrier frequency of $200 \mathrm{MHz}$. Here, the performance for lower symbol rates is poorer due to phase noise since lower symbol rate symbols are transmitted over a longer period of time and therefore experience additional phase noise. ${ }^{6}$ The inset shows the constellation of the received signals for a symbol rate of 10 MBaud, where the phase noise when unlocked is increased compared with the previous result due to the lower symbol rate.

\section{4 | CONCLUSIONS}

This article has presented the use of RTD based photodetectors for optical communications. Modulated optical signals were used to excite the RTD-PDs and successful data experiments were conducted up to $80 \mathrm{Mbit} / \mathrm{s}$. As well as simple modulation formats, it was shown that these oscillators can respond to more complex data signals up to $100 \mathrm{MBaud}(200 \mathrm{Mbit} / \mathrm{s})$ and work is on-going to increase data rates accordingly.

\section{ACKNOWLEDGMENTS}

This work was performed as part of the iBROW project which has received funding from the European Union's Horizon 2020 Research and Innovation Programme under grant agreement No. 645369.

\section{ORCID}

Scott Watson (ID) https://orcid.org/0000-0002-9235-9242

Joana Tavares (D) https://orcid.org/0000-0002-7666-3738

\section{REFERENCES}

[1] Kleine-Ostmann T, Nagatsuma T. A review on terahertz communications research. J Infrared Millim Terahertz Waves. 2011;32(2): 143-171.

[2] Maekawa T, Kanaya H, Suzuki S, Asada M. Oscillation up to 1.92 $\mathrm{THz}$ in resonant tunnelling diode by reduced conduction loss. Appl Phys Express. 2016;9(2):024101.

[3] Oshima N, Hashimoto K, Suzuki S, Asada M. Wireless data transmission of $34 \mathrm{Gbit} / \mathrm{s}$ at a $500-\mathrm{GHz}$ range using resonant-tunnellingdiode terahertz oscillator. Electron Lett. 2016;52(22):1897-1898.

[4] Romeira B, Pessoa LM, Salgado HM, Ironside CN, Figueiredo JML. Photo-detectors integrated with resonant tunneling diodes. Sensors. 2013;13:9464-9482.

[5] Tavares JS, Pessoa LM, Figueiredo JML, Salgado HM. Analysis of resonant tunnelling diode oscillators under optical modulation. In: Proceedings of the ICTON, PDP41, Girona; 2017.

[6] Khanzadi MR, Kuylenstierna D, Panahi A, Eriksson T, Zirath H. Calculation of the performance of communication systems from measured oscillator phase noise. IEEE Trans Circuits Syst I: Regul Pap. 2014;61(5):1553-1565.

How to cite this article: Watson S, Zhang W, Tavares J, et al. Resonant tunneling diode photodetectors for optical communications. Microw Opt Technol Lett. 2019;61:1121-1125. https://doi.org/10. 1002/mop.31689 